Instituto Internacional de Investigación y Desarrollo Tecnológico Educativo INDTEC, C.A.

DOI: https://doi.org/10.29394/Scientific.issn.2542-2987.2020.5.18.17.323-344

OAI-PMH: http://www.indteca.com/ojs/index.php/Revista Scientific/oai

Ensayo Original / Original Essay

\title{
Desafío del Siglo XXI en la educación: dando saltos del TIC-TAC al TEP
}

\author{
Autoras: Marcia Geoconda González González \\ Universidad Católica de Cuenca, UCACUE \\ marcia.gonzalez@psg.ucacue.edu.ec \\ Azogues, Ecuador \\ https://orcid.org/0000-0002-2432-1015 \\ Mónica Carolina Ojeda Chimborazo \\ Universidad Católica de Cuenca, UCACUE \\ monica.ojeda@psg.ucacue.edu.ec \\ Azogues, Ecuador \\ https://orcid.org/0000-0003-1242-978X \\ Paola Cecilia Pinos Coronel \\ Universidad Católica de Cuenca, UCACUE \\ paola.pinos@psg.ucacue.edu.ec \\ Azogues, Ecuador \\ https://orcid.org/0000-0003-4770-9116
}

\section{Resumen}

Es evidente que el proceso educativo ha sufrido un cambio significativo debido a la incursión de las Tecnologías de la Información y Comunicación (TIC); Tecnología del Aprendizaje Cooperativo (TAC), razón por la cual este trabajo investigativo está enfocado al uso, sus ventajas y desventajas. La importancia que tienen la implementación de las TIC desde edades tempranas para lograr a futuro un TAC y Tecnologías del Empoderamiento y la Participación (TEP), como un reto en la educación, con trabajo colaborativo, cooperativo, entre otras, y obtener un aprendizaje significativo con estudiantes inventores, creativos-reflexivos lo que hace falta para esta sociedad. Además, el rol del maestro y del estudiante cambia al usar correctamente las Tic y redes sociales como herramientas manejadas por todos, modificando de esta manera la educación tradicional a una educación interactiva a través de WhatsApp involucrando a los padres o representes legales al triángulo educativo dentro de este proceso. Por otro lado, se analiza las brechas existentes que limitan al uso de las TIC en una institución, siendo internas y externas. El docente del siglo XXI es pieza clave para cambiar la educación en tiempos de emergencia como lo estamos viviendo y desarrollar el pensamiento crítico desde edades tempranas.

Palabras clave: educación; tic; profesor; cooperativo; colaborativo.

Cómo citar este ensayo:

González, M., Ojeda, M., \& Pinos, P. (2020). Desafío del Siglo XXI en la educación: dando saltos del TIC-TAC al TEP. Revista Scientific, 5(18), 323-344, e-ISSN: 2542-2987. Recuperado de: https://doi.org/10.29394/Scientific.issn.2542-2987.2020.5.18.17.323-344

Fecha de Recepción: 11-04-2020
Fecha de Aceptación: 20-08-2020
Fecha de Publicación: 05-11-2020 
Instituto Internacional de Investigación y Desarrollo Tecnológico Educativo INDTEC, C.A.

DOI: https://doi.org/10.29394/Scientific.issn.2542-2987.2020.5.18.17.323-344

OAI-PMH: http://www.indteca.com/ojs/index.php/Revista_Scientific/oai

Ensayo Original / Original Essay

\title{
Challenge of the XXI Century in education: making leaps from ICT-TAC to TEP
}

\begin{abstract}
It is evident that the educational process has undergone a significant change due to the incursion of Information and Communication Technologies (ICT); Cooperative Learning Technology (TAC), which is why this research work is focused on use, its advantages and disadvantages. The importance of the implementation of ICT from an early age to achieve a TAC and Technologies of Empowerment and Participation (TEP) in the future, as a challenge in education, with collaborative and cooperative work, among others, and obtain significant learning with inventive, creative-reflective students what is needed for this society. In addition, the role of the teacher and the student changes when using ICT and social networks correctly as tools managed by all, thus modifying traditional education to an interactive education through WhatsApp involving parents or legal representatives of the educational triangle within of this process. On the other hand, the existing gaps that limit the use of ICT in an institution are analyzed, being internal and external. The teacher of the 21st century is a key piece to change education in times of emergency as we are living it and to develop critical thinking from an early age.
\end{abstract}

Keywords: education; ict; teacher; cooperative; collaborative.

How to cite this essay:

González, M., Ojeda, M., \& Pinos, P. (2020). Challenge of the XXI Century in education: making leaps from ICT-TAC to TEP. Revista Scientific, 5(18), 323-344, e-ISSN: 2542-2987. Recovered from: https://doi.org/10.29394/Scientific.issn.2542-2987.2020.5.18.17.323-344

Date Received: 11-04-2020
Date Acceptance:

20-08-2020
Date Publication:

05-11-2020 


\section{Introducción}

La nueva era digital ha transformado la educación. El constante uso de las Tecnologías de la Información y Comunicación (TIC) se ha transformado en un recurso indispensable en el aula, cada vez es necesario implementar nuevos métodos y técnicas que permitan al docente dar un significado educativo de alto accionar en las aulas clase, es así, como la educación gira entorno a la tecno pedagogía cuyo único objetivo es garantizar el conocimiento como aporte significativo al entorno y así llegar a un progreso de la sociedad.

La incorporación tecnológica se ha vuelto prioritaria en la educación en donde los docentes enfrentan un nuevo desafío, ya que, ellos están obligados a preparar institucionalmente a los estudiantes de manera holística garantizando la integración satisfactoria en la sociedad.

Es notable la diferencia que existe en el manejo tecnológico entre docentes y estudiantes, por lo que los docentes se convierten en migrantes a este medio de adaptación con nativos tecnológicos, se enfrentan a un proceso complejo que refleja su capacidad de aplicar las diferentes herramientas, recursos, materiales, etc. que intervienen en el sumario de ejercer eficazmente en la gamificación de contextos educativos que surten.

Esto implica un cambio en los hábitos y materiales educativos, comprender a los nativos digitales (estudiantes) se torna un verdadero desafío por parte de las migrantes digitales (docentes); las actividades académicas ya no son rígidas y es necesario que el docente proponga nuevos métodos e instrumentos innovadores que despierten el aprendizaje del estudiante para la vida.

Es así como la tecnología desde tiempo atrás se ha convertido en el motor del desarrollo del conocimiento del hombre, siendo primordial en estos procesos, es así como los docentes al ser entes principales de educación conviene que estén capacitados para convertir la tecnología en un instrumento a favor, con el que, despertarán el interés de sus estudiantes. 
Es notable la reacción adversa de los docentes al estar cómodamente en un ambiente que manejan un mismo texto o libro durante varios años; y de un momento a otro existe información digitalizada al 100, según Sierra, Bueno y Monroy (2016): en una investigación realizada, da a conocer que los materiales más usados son los tradicionales, de esta manera los resultados que se obtienen son muy similares a los reflejados sin el uso de TIC.

Las Tecnologías del Aprendizaje y del Conocimiento o Tecnologías del Aprendizaje Cooperativo (TAC), para Zulaica y Villagómez (2019): se presentan al servicio de la educación, dando un giro importante en el mismo, en el que se abren caminos que mejoran el proceso de enseñanza-aprendizaje considerando la relevancia de los contenidos educativos digitales y la integración de estos en el aula, se trata entonces de lograr la instrucción y actualización docente para el buen uso de estas herramientas

El profesor TAC usa las TIC facilitando el aprendizaje, esto no implica mayor cambio de metodología, al contrario, requiere el uso de las metodologías activas que se usa a diario en el aula y su adaptabilidad con las TIC, es decir, durante el proceso interactivo que se usa basado en la comunicación lo cual nos da como resultado un aprendizaje satisfactorio y enriquecido las TAC hacen un aporte avanzado posibilitando el conocimiento garantizado, con esta se crea, se comparte, se difunde, incluso se presentan diferentes opiniones no solo dentro del aula, de la institución, provincia o austro, sino, alrededor del mundo y en un tiempo real.

Ecuador es un país que establece en su currículo el uso de las TIC en el contexto educativo, es por eso que, la evolución de la tecnología, pedagogía y los constantes cambios demandan una formación docente en integración tecnológica.

En un estudio realizado en la ciudad de Ambato por Mayorga (2020a): esclarece en su investigación que existe un alto conocimiento en TIC, pero en TAC y TEP el porcentaje es menor, además menciona que de toda la población 
ecuatoriana un $25 \%$ de la población tiene como profesión la docencia por lo que es de suma importancia realizar un enfoque en las tres dimensiones: TIC, TAC Y TEP para garantizar la educación de calidad que aporte a la solución de problemas. Los docentes conocen sobre las TIC, pero no lo aplican impidiendo dar los siguientes pasos. Una constante capacitación que permita aplicar y usar enfoca una educación holística que demanda la sociedad hoy en día.

A partir de este punto se considera importante dar a conocer las TAC como instrumento educativo, ya que es de gran utilidad y favorecimiento para el aprendizaje de los estudiantes. Analizar el contexto es de suma importancia para no ocasionar un fracaso de implementación a falta de recursos como menciona Luque (2016): existen limitaciones como la falta de formación docente, la capacidad de los alumnos de diferenciar verdadera información de la múltiple que se encuentra en la web, entre otras que limita el uso adecuado de las TAC en el aula.

\section{Desarrollo}

\subsection{La Tecnología Revoluciona la Educación}

El progreso de la sociedad ha sido marcado cada vez más rápido por las líneas de tiempo, avanza progresivamente sin descanso; no es ignorada entonces la tecnología y el privilegio que ha tomado en estos últimos momentos. Estar capacitado para una competencia digital es una labor fundamental para el docente, de acuerdo con Villarreal-Villa, García-Guliany, Hernández-Palma y Steffens-Sanabria (2019): la alfabetización digital es un primer paso que dará competencia íntegra donde se analicen y se estudien las competencias necesarias para un correcto desenvolvimiento.

Es así como incluir las TIC desde los primeros años de educación básica podría ser de suma importancia y beneficencia; en un mundo donde la era digital demanda actualización y análisis de las nuevas tecnologías. De 
acuerdo a la investigación realizada por Bustamante y De Lima (2020): los docentes de educación inicial desde un enfoque de 5 competencias como tecnológica, comunicativa, pedagógica, investigativa y gestión, el nivel que refleja mayor uso por parte de ellos es la investigativa, lo cual refleja que es necesario y aún falta una integración TAC y TEP desde los primeros años de educación.

Es muy común utilizar hoy en día la tecnología en varios procesos de la educación desde su planificación, evaluación y desarrollo en donde aporta un sinfín de beneficios que mejoran la eficiencia y productividad si se emplea de manera correcta, incluso sirve como herramienta de seguimiento a los estudiantes. Está presente en todo momento de la planificación educativa: inicio, desarrollo y avaluación tanto de docentes como de estudiantes entorno a los contenidos específicos de cada tema tratado. Es necesario aprovecharlas con dirección a un aprendizaje significativo de parte a parte y sobre todo cooperativo, en un estudio de caso realizado por Latorre, Castro y Potes (2018a): muestra la importancia de fortalecer, renovar y adecuar los contenidos tecnológicos ya que las prácticas educativas se apoyan en diferentes estrategias pedagógicas para garantizar el aprendizaje.

Un país se basa en el desarrollo y progreso de los pueblos y se considera a la educación como uno de los ámbitos más importantes para este proceso, en el cual se brinde su apoyo, facilitando equipos y capacitaciones para la educación en zonas rurales y urbanas donde se prioriza lo rural para garantizar que el servicio, obteniendo como beneficio disminuir el analfabetismo digital.

El Sistema Integral de Tecnologías para la Escuela y la Comunidad (SITEC) propone implementar en los establecimientos educativos fiscalesrurales y urbano-marginales de computadoras, de pizarras digitales y proyectores, con el objetivo de acortar la gran brecha digital en la educación, para de esta manera suprimir un poco el analfabetismo digital. Además, 
promueven capacitaciones, diseñan y ejecutan proyectos acerca del uso de TIC en clases con el objetivo apoyar a este proceso enseñanza aprendizaje del siglo XXI.

Con este nuevo giro que la educación enfrenta, incorporar tecnología colaborativa en los procesos educativos se ha convertido en un verdadero reto, por lo que, uno de los pasos más importantes, como dice Monguillot, González y Guitert (2017): es realizar un proceso reflexivo en los docentes a partir de las diferentes experiencias en el aula para evaluar y generar nuevas estrategias.

En las manos de los docentes está el futuro de la sociedad, por lo que es importante educar con una visión a la solución de problemas y un desenvolvimiento eficaz en la vida; implementar TAC desde los primeros años de educación beneficia al surgimiento de adaptación al cambio de planteamiento didáctico que demanda el currículo. Como lo manifiesta Mayorga (2020b): se promueve una educación para el pensamiento creativo con ayuda de las TIC, en donde los docentes actúan como guías en el camino de los estudiantes.

La dotación e incorporación de herramientas tecnológicas que brinden un aprendizaje significativo en esta era digital apoyará a la formación integral de los estudiantes para ello es indispensable replantear el currículo de acuerdo al contexto y garantizar la competitividad del docente frente a este nuevo reto de educación siglo XXI. Es así como todas unas comunidades educativas deben evaluar periódicamente las técnicas empleadas en el proceso de enseñanza-aprendizaje.

El docente preparado en este tema hace uso de la tecnología en sus diferentes aspectos y categorías dando saltos pequeños y bien utilizados del TIC al TAC y con metodologías bien combinadas y adaptadas direccionadas a un aprendizaje colaborativo, cooperativo, basado en problemas, entre otras que integran las metodologías activas para lograr la creación de conocimientos cuyo objetivo es aprender lo mayormente posible y de mejor calidad. 
La tecnología entonces, ayuda a la innovación educativa en donde el estudiante recoge, investiga y analiza la información obtenida en la red y el docente se convierte en un auxiliar cognitivo, un guía que cuestiona al estudiante para lograr un aprendizaje significativo y creativo. De acuerdo con Latorre, Castro y Potes (2018b): es así entonces como las TIC, al incorporarse en la educación y al ser aplicadas dan un salto importante a las TAC, y cuando se logra un aprendizaje práctico, significativo, creativo se da un paso más a las Tecnologías del Empoderamiento y la Participación (TEP).

Las TIC y las TAC bien usadas nos pueden garantizar el potencial estudiantil en una interacción y colaboración entre sí, como creadores de contenido en un mundo virtual, sin limitarlos a ser pasivos, simples observadores. Se aprovecha la interacción entre docentes y alumnos para lograr un aprendizaje aumentado, de esta manera los estudiantes guiados por el docente logran un aprendizaje permanente, aprovechando la información de internet, rescatando recursos, información y estimulo permanente por el aprendizaje.

En consonancia con lo expuesto por Martínez, Cascales-Martínez y Gomariz-Vicente (2017a): donde dan a conocer las tecnologías como uso didáctico que beneficia el aprendizaje basándose en la resolución de problemas aplicando el aprendizaje colaborativo concluyen que la tecnología bien aplicada promueve innovación educativa por la posibilidad a un ambiente propicio de aprendizaje. Incorporar la tecnología en las aulas demanda capacitaciones e información actual y constante por lo que es necesario estar activo y presto a aprender. Por otro lado, señalan el gran beneficio que provoca la tecnología en los estudiantes ya que en este estudio realizado se observa el rol activo y participativo, ofreciéndoles flexibilidad, experimentación y manipulación, es así entonces como de manera indirecta está presente las TAC encaminadas a las TEP.

Ante lo mencionado, la incursión y el apoyo de la tecnología fusionada 
con metodologías activas despierta el interés del estudiante por ser él quien vaya descubriendo e interiorizando su propio aprendizaje para ello los docentes serán una pieza estratégica en este proceso de aprendizaje como guías, coordinadores, o más bien como mediadores en el camino del aprendizaje significativo y real para los estudiantes el mismo que demandará por parte del docente una preparación adecuada en metodología y uso de aplicaciones tecnológicas llegando a ser un docente creativo e innovador.

De esta manera es evidente que uso de las TIC y la importancia que se da en el proceso educativo ha dado un gran progreso a la sociedad marcado cada vez más rápido en las líneas del tiempo y porque no en la vocación de un docente que es quién tiene en sus manos la responsabilidad de un futuro mejor identificando oportunidades y problemas existentes en la utilización pedagógica.

Con todo lo dicho anteriormente las TIC solo son una herramienta a favor de la educación, pero el docente es quien le dará el uso correcto de estas aplicaciones al servicio para llegar al TAC donde se logra el objetivo pedagógico planteado obteniendo un aprendizaje significativo en los estudiantes y dar el gran salto al TEP que es llevar a la práctica del conocimiento adquirido para la solución de problemas dentro de la sociedad. De esta convicción nace la idea que incluir TIC, TAC y TEP desde los primeros años de educación básica es primordial para enfrentar a esta nueva era digital en la cual las competencias son las grandes demandas del futuro.

\subsection{El gran salto de las TIC y TAC al TEP a través de las redes sociales}

En las múltiples interacciones que la sociedad presenta, existe la interacción de participantes con varias preferencias, estas funcionan a modo de organización sencilla, actual y de menor tiempo posible. Es así como las redes sociales toman las riendas principales del camino y que conforme a Ávila-Toscano (2012): las redes sociales se tratan de un entramado social 
considerándolo dentro de una estructura social, donde la intención de interacción es la misma, información y comunicación entre personas.

En la mayoría de las instituciones educativas se ha regularizado la prohibición del uso de redes sociales, pero lo que no nos hemos puesto a analizar son las ventajas que esta conlleva. Las redes sociales son una serie de aplicaciones y sitios tecnológicos que permite la interacción con otras personas intercambiando información de valor o interés común sin una jerarquía establecida o limitantes físicos. Entre los más comunes y conocidos por todo el mundo están: Facebook, WhatsApp, Twitter, Snapchat o Instagram; sin embargo, este término también es usado para analizar las interacciones entre sociedades, individuos, grupos, entre otros; lo que nos lleva a un aprendizaje TEP.

La era digital de hoy en día nos ha llevado a utilizar de manera intuitiva las redes sociales y en el caso de los estudiantes la tecnología como instrumentos básicos dentro de la sociedad, sobre todo en los nativos digitales que sus conexiones se basan fundamentalmente en un texto escrito en las diferentes redes sociales, siendo Facebook y WhatsApp las más usadas, dichas redes han repercutido también en la educación, se ha visto la necesidad de incorporarlos no solo para tareas administrativas y de información a padres de familia, representantes legales y entre docentes mismo si no de docentes como herramientas e instrumentos para interactuar con información y crear entornos de aprendizaje colaborativo.

Las TEP pretenden emerger las ventajas que tienen las redes sociales y el buen uso de las mismas, conforme a Marín-Díaz y Cabero-Almenara (2019): se analiza la incorporación de las redes sociales en la enseñanza, su aspecto positivo y también lo negativo en donde concluye que dicha herramienta es básica en la sociedad hoy en día repercutiendo en el crecimiento exponencial en los entornos de formación.

Un constante apoyo donde intervenga la colaboración como eje 
fundamental, el compartir conocimientos, el interés por aprender y enseñar con una constante participación y un pensamiento crítico y reflexivo en el momento de buscar información, mejora de comunicación, realización de trabajos y relaciones interpersonales e intrapersonales son algunas ventajas de las redes sociales dentro del contexto de educación. Menciona Alvarado, Ochoa, Ronquillo y Sánchez (2019): que las ventajas de las redes sociales crean como consecuencia una muestra novedosa y actual en el que se presentan diferentes elementos que influyen directamente en el acogimiento de aplicaciones actuales y redes sociales con un fin educativo manejando una perspectiva original e innovadora.

Una de las redes sociales de mayor impacto hoy en día es WhatsApp, tanto docentes, alumnos, padres de familia, representantes legales, directivos entre otros usan y tienen este aplicativo, que en muchos de los casos ha reemplazado las Ilamadas telefónicas. Es necesario recordar a Suárez (2018): quien destaca a WhatsApp como la herramienta que más se ha usado con fines pedagógicos, algunas ventajas que menciona en su artículo son: fluidez, comunicación efectiva e intercambio de información.

Varios estudios y trabajos destacan la actitud positiva de WhatsApp y motivan a que la usen como herramienta de apoyo en el proceso de enseñanzaaprendizaje; ahora bien, tomando desde este punto de vista se sabe que es una herramienta de alto impacto y de gran ayuda, pero, el tema de usos de redes sociales en la educación está limitado. Muchas instituciones prohíben el uso de un dispositivo electrónico en las aulas clase, y otros que permiten el uso hace énfasis en la prohibición de uso de redes sociales.

Si bien es cierto que este tipo de herramientas no se pueden usar en el aula clase, pero se pueden usar como herramienta de refuerzo fuera de él, el docente toma fotos de los apuntes en clase y puede enviar al grupo de estudiantes como respaldo de información o refuerzo de la hora clase, esto dentro de un rango de edades determinadas en las que un teléfono celular sea 
adquirible. Siendo así, ¿cómo se trabaja entonces con los primeros años de básica, donde un teléfono o un dispositivo con esta herramienta no sea parte del niño o niña?. Hemos mencionado ya, que la comunidad educativa la integran todos: docentes, directivos, alumnos y padres de familia o representantes legales, por lo que, si un niño no tiene un dispositivo su padre, madre, o representante lo dispone, y es ese el uso que se da; el docente envía sus refuerzos o aportes al proceso de aprendizaje a través de este medio, o en su defecto, se suele trabajar con la directiva del aula, los refuerzos son enviados al presidente de padres de familia de grado o curso y este a su vez envía o comparte la información al grupo de padres de familia del aula. Incluso la mayoría de docentes usa esta herramienta para compartir fotos de los trabajos que sus representados realizan en el aula clase.

El uso de los grupos de WhatsApp creados entre familias y docentes sin lugar a duda fomenta la interacción, de este modo, Martínez, CascalesMartínez y Gomariz-Vicente (2017b): en un análisis del uso de esta herramienta entre familias y docentes indica en sus resultados que no se considera útil este medio a la hora de fomentar participación familiar, si no como un medio para mantener la conectividad. es muy clara la evidencia de este medio al hacer presencia cuando se inicia y establece relaciones. Varios centros educativos se mantienen al margen de esta dinámica, limitándose a dar información y a regular el tipo de publicaciones.

En este sentido, Jiménez (2020): en su análisis científico del uso de la red social WhatsApp en los estudiantes, concluye que es una herramienta de soporte académico tanto para docentes como para estudiantes en donde logran desenvolverse en los entornos virtuales que están en auge hoy en día, logrando una integración, personalización, gestión, creatividad e interacción. De igual forma, funciona con la comunidad educativa, en este caso los padres de familia o representantes legales de los niños y niñas que tienen acceso limitado a los dispositivos electrónicos. 
La educación actual demanda la incorporación de las TIC, y para un conocimiento las TAC y de las redes sociales como instrumentos $y$ herramientas base en el proceso de enseñanza-aprendizaje, concluyen como TEP mismo reta al docente por ser un migrante tecnológico frente a una población nativa tecnológica a incorporar de manera gradual y consciente en las actividades educativas. En un análisis de Tapia-Repetto, Gutierrez y Tremillo-Maldonado (2019): respecto al uso de WhatsApp frente al EVA refleja una interacción mayor por parte de los estudiantes, por la facilidad de comunicación y tiempo que requiere el uso de esta aplicación, por ser instantánea y de fácil uso. Un $57 \%$ de los estudiantes reflejaron que WhatsApp es superior al EVA, un $33 \%$ les dio igualdad a los instrumentos y solamente un $10 \%$ consideró a EVA como el mejor de los instrumentos. De la misma manera la interacción de docentes y estudiantes revela un índice alto, un $70 \%$ otorga como una mejor herramienta de comunicación, esta se relaciona además con los trabajos en grupo que se realizan.

El impacto que presenta la comunicación de WhatsApp, es una herramienta virtual que ayuda al trabajo colaborativo no solo entre estudiantes, estudiantes-docentes, sino también entre docentes. En esa misma línea, Monguillot, González y Guitert (2017a): muestra que la experiencia que los docentes han palpado en el diseño y manejo, al momento de efectuar y valorar ámbitos de aprendizaje, mismas que son medidas por el TAC, además el gran valor del uso de la herramienta WhatsApp en un uso permanente de cooperación docente.

De esta manera se ve reflejada la utilidad de esta herramienta fomentando la creación y construcción de conocimientos, en referencia al tema, Monguillot, González y Guitert (2017b): considera el WhatsApp como una herramienta positiva e innovadora que coopera durante el proceso de enseñanza-aprendizaje, es importante tomarla en cuenta para una implementación, diseño y evaluación de situaciones, proyectos y varios 
entornos de aprendizaje en donde se ve reflejada la colaboración.

\subsection{Reto del TIC, TAC y TEP en la educación actual}

Durante todo el proceso hemos podido observar y analizar la gran ventaja y que tiene el uso de las TIC en educación y el poder que lleva consigo, pero existen varios factores que impiden el uso correcto de las mismas, y no es precisamente la falta de capacitación del docente par el uso de las mismas, más bien, se tratan de factores internos y externos que según Martinez y Guillén-Guerrero (2019a): los externos no están relacionados con una responsabilidad directa del docente, algunos de ellos son: falta de centros informáticos en las instituciones, lo que conlleva la escases de computadoras para docentes y alumnos, ya que, en el análisis que realizó existían pero estaban a cargo de los técnicos.

La ausencia de internet es otro factor en contra, si bien es cierto que las instituciones educativas existe el alcance de una red wifi muchas de las veces está al alcance solamente de los directivos, mismos que limitan el uso de estos a los mismos docentes y en su defecto a los alumnos, para lograr una cobertura en varios casos los docentes pagan de su bolsillo lo cual no es satisfactorio y mucho menos se encuentra colaboración de todos, siendo esta red limitada para el uso docente sin lograr cobertura estudiantil.

Otro factor que mencionan es la escasez de programas nacionales de capacitación y entrenamiento en informática, por este lado el gobierno nacional ofrece cursos constantes pero no son obligatorios, el sistema de redes de docentes oferta cursos de capacitación pero estos son limitados, el acceso es en la mayoría de veces para los docentes fijos dentro del ministerio sin considerar el gran número de docentes contratados que están al servicio de la educación, esto sin mencionar el abordaje que emplea para ubicar a los docentes en áreas que no les corresponden o que tienen poco o nulo conocimiento obligando a la autoeducación en una responsabilidad bien 
llevada o en su contrariedad al fracaso del aprendizaje por una comodidad docente.

Por otro lado, los factores internos, mencionan Martinez y GuillénGuerrero (2019b): son aquellos que se relacionan directamente con la responsabilidad del docente, entre estos tenemos, falta de capacitación, escasez de experiencia y conocimientos en el uso de las TIC, y sobre todo la resistencia al cambio. Hemos mencionado ya que la mayoría de docentes son migrantes tecnológicos tratando de adaptarse en un mundo lleno de nativos digitales, muchas de las veces el miedo al rechazo por parte de los estudiantes es un factor en contra, pero es necesario considerarlos como una herramienta de apoyo y romper las barreras, romper el estereotipo que el docente sabe todo, cuando estamos en un proceso de enseñanza-aprendizaje porque se aprende en conjunto, la vida es un constante aprendizaje, el ser humano no deja de aprender hasta que muere, pues esto deben considerar los docentes que están al frente para aprender de los estudiante cosas nuevas y usarlas a favor de la educación.

Encontramos limitantes, y es necesario empezar con el cambio e incluir la tecnología desde los primeros años de educación básica. Implementar nuevas metodologías o adaptarlas correctamente es de suma importancia para lograr TAC en educación, para llegar a este punto holístico es necesario la integración de nuevos idiomas, nativos culturales como el quechua o internacionales como el inglés, tal como menciona Martínez, CascalesMartínez y Gomariz-Vicente (2017c): es necesario desarrollar tecnologías con una metodología plurilingüe desde muy anticipadas edades.

\section{Conclusiones}

Definitivamente la educación está en revolución conjuntamente con el uso de Tic y Tac, una educación de calidad integral del estudiante, la misma que garantice un conocimiento significativo, reflexivo, personas capaces de 
solucionar los problemas que se presentarán en la educación del siglo XXI. Será un desafío tanto de estudiantes como de docentes, pero si tiene la capacitación necesaria y las ganas de cambiar a esta educación tradicional se lograrán obtener buenos resultados a nivel educativo.

De acuerdo a este artículo la tecnología ocupa ya un lugar primordial en el sistema educativo, más en esta etapa de crisis sanitaria, pero se ha observado que hay una brecha enorme en el uso correcto de las diferentes aplicaciones que nos brinda las TIC para lograr un aprendizaje significativo en los estudiantes (TAC). Por ende, los docentes están cumpliendo su papel de educar a través de las plataformas o aplicaciones y de esta manera no perder la interacción docentes- estudiantes y padres de familia; en tal sentido, los maestros están facilitando el proceso aprendizaje a través de innovaciones tecnológicas dejando de ser migrantes tecnológicos.

Por todo lo expuesto en este artículo, se puede recalcar que el uso de las TIC se debe impartir desde las primeras edades para luego dar un avance muy significativo en su aprendizaje, al ser el estudiante quién construya su conocimiento mediante estrategias como el trabajo cooperativo, colaborativo. Estas estrategias definitivamente facilitan la interacción entre los estudiantes y profesores hasta llegar a un consenso y a un aprendizaje cognitivosignificativo TAC.

El uso de las TIC en las planificaciones de los docentes ya es un requisito para el desarrollo de su clase, razón por la cual, es necesario conocer el uso de las diferentes aplicaciones que nos brinda la tecnología para llegar a un aprendizaje de calidad dentro y fuera de las aulas. Las TIC al ser utilizadas por el profesor de una manera eficaz integrando estrategias activas como el trabajo colaborativo, cooperativo entre otras, garantiza el cambio de una clase magistral y tradicional a una clase interactiva donde el estudiante es el artífice de su propio aprendizaje guiado por el profesor.

En tal sentido el docente necesita capacitación o auto capacitación si 
así se puede llamar para vencer la gran brecha que se tiene a cerca de la Tecnología incursionada en el proceso educativo y el uso correcto de las diferentes aplicaciones, plataformas para dejar de ser migrantes tecnológicos e ir de la mano con la evolución de la educación y la tecnología y por qué no dar el gran salto de TIC al TAC y cumplir con el objetivo de una educación integral y de calidad que es la exigencia de hoy en día a pesar que dentro de una misma institución o centro educativo se tiene limitantes.

Los estudiantes deben disfrutar de este proceso educativo, por esta razón las redes sociales es una herramienta que nos brinda la tecnología, entonces el docente debe ser creativo para implementar el uso de ellas e innovar de esta forma la manera de impartir conocimientos haciendo uso de los que más disfrutan los estudiantes.

En conclusión, el desafío esta dado, ser profesores del siglo XXI no será tarea fácil, puesto que los docentes deberán estar preparados para asumir este reto y potenciar sus habilidades en el manejo de la tecnología desde tempranas edades ya que el docente es un arma potencial en el desarrollo del proceso educativo.

\section{Referencias}

Alvarado, E., Ochoa, M., Ronquillo, G., \& Sánchez, M. (2019). Importancia y uso de las redes sociales en la educación. Recimundo: Revista Científica Mundo de la Investigación y el Conocimiento, 3(2), 882-893, e-ISSN: 2588-073X. Recuperado de:

http://recimundo.com/index.php/es/article/view/481

Ávila-Toscano, J. (2012). Redes sociales y análisis de redes. Aplicaciones en el contexto comunitario y virtual. ISBN: 978-958-57406-0-0. Barranquilla, Colombia: Corporación Universitaria Reformada.

Bustamante, L., \& De Lima, K. (2020). Nivel de competencias TIC de docentes de preescolar. Infancias Imágenes, 19(1), 83-90, e-ISSN: 
1657-9089. Recuperado de:

https://revistas.udistrital.edu.co/index.php/infancias/article/view/13936 Jiménez, O. (2020). Uso de Red Social WhatsApp por los estudiantes del tercer y cuarto grado de educación secundaria en la Institución Educativa Libertador San Martín - San Borja, Lima - 2019. Lima, Perú: Universidad Católica Los Ángeles de Chimbote.

Latorre, E., Castro, K., \& Potes, I. (2018a,b). Las TIC, las TAC y las TEP: innovación educativa en la era conceptual. ISBN: 978-958-5511-439. Bogotá, Colombia: Universidad Sergio Arboleda.

Luque, F. (2016). Las TIC en educación: caminando hacia las TAC. 3C TIC: Cuadernos de desarrollo aplicados a las TIC, 5(4), 55-62, e-ISSN: 22546529. Recuperado de: http://dx.doi.org/10.17993/3ctic.2016.54.55-62 Martínez, I., Cascales-Martínez, A., \& Gomariz-Vicente, M. (2017a,b,c). Grupos de WhatsApp en familias de Educación Infantil y Primaria. Relatec: Revista Latinoamericana de Tecnología Educativa, 16(2), 239255, e-ISSN: 1695-288X. Recuperado de:

https://doi.org/10.17398/1695-288X.16.2.239

Martinez, O., \& Guillén-Guerrero, G. (2019a,b). Tecnologías de la Información y la Comunicación e Innovación Tecnológica en la Educación Básica. Risti, (E18), 1-13, e-ISSN: 1646-9895. Recuperado de: http://www.risti.xyz/issues/ristie18.pdf

Marín-Díaz, V., \& Cabero-Almenara, J. (2019). Las redes sociales en educación: desde la innovación a la investigación educativa. RIED: Revista Iberoamericana de Educación a Distancia, 22(2), 25-33, eISSN: 1390-3306. Recuperado de:

http://dx.doi.org/10.5944/ried.22.2.24248

Mayorga, M. (2020a,b). Conocimiento, Aplicación e Integración de las TIC -TAC y TEP por los Docentes Universitarios de la Ciudad de Ambato. Revista Docentes 2.0, 9(1), 5-11, e-ISSN: 2665-0266. 
Recuperado de: https://doi.org/10.37843/rted.v9i1.101

Monguillot, M., González, C., \& Guitert, M. (2017). El WhatsApp como herramienta para la colaboración docente. EmásF: revista digital de educación física, (44), 56-62, e-ISSN: 1989-8304. Recuperado de: https://dialnet.unirioja.es/servlet/articulo?codigo $=5807534$

Sierra, J., Bueno, I., \& Monroy, S. (2016). Análisis del uso de las tecnologías TIC por parte de los docentes de las Instituciones educativas de la ciudad de Riohacha. Omnia, 22(2), 50-64, e-ISSN: 1315-8856. Recuperado de: https://www.redalyc.org/articulo.oa?id=73749821005 Suárez, B. (2018). WhatsApp: su uso educativo, ventajas y desventajas. Revista de Investigación en Educación, 16(2), 121-135, e-ISSN: 21723427. Recuperado de:

http://reined.webs.uvigo.es/index.php/reined/article/view/342

Tapia-Repetto, G., Gutierrez, C., \& Tremillo-Maldonado, O. (2019). Nuevas tecnologías en educación superior. Estudio de percepción en estudiantes acerca del uso de WhatsApp y Entornos Virtuales de Aprendizaje (Plataforma Moodle). Odontoestomatología, XX(33), 3743, e-ISSN: 1688-9339. Recuperado de:

https://dx.doi.org/10.22592/ode2019n33a5

Villarreal-Villa, S., García-Guliany, J., Hernández-Palma, H., \& SteffensSanabria, E. (2019). Competencias Docentes y Transformaciones en la Educación en la Era Digital. Formación Universitaria, 12(6), 314, e-ISSN: 0718-5006. Recuperado de:

https://dx.doi.org/10.4067/S0718-50062019000600003

Zulaica, H., \& Villagómez, A. (2019). La innovación tecnológica (tic y tac) en una escuela de educación primaria. RILCO: Revista de Investigación Latinoamericana en Competitividad Organizacional, 1(2), 1-9, e-ISSN: 2659-5494. Recuperado de:

https://www.eumed.net/rev/rilco/02/tic-tac.html 


\section{Marcia Geoconda González González}

e-mail: marcia.gonzalez@psg.ucacue.edu.ec

Nacida en Guapán, Azogues, Ecuador, el 7 de diciembre

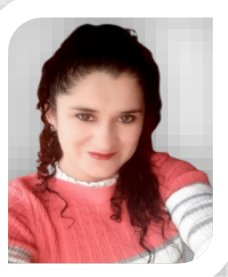

del año 1974. Los estudios primarios los realice en la Unidad Educativa Particular "La Providencia", Azogues; los segundarios en el Colegio Luis Cordero; y los estudios superiores en la Universidad de Cuenca (UCUENCA), obteniendo el título de Licenciada en Ciencias de la Educación especialidad Lengua Inglesa; actualmente Laboro en la Unidad Educativa Particular "La Providencia", Azogues desde hace 20 años como docente de inglés. 


\section{Mónica Carolina Ojeda Chimborazo e-mail: monica.ojeda@psg.ucacue.edu.ec}

Nacida en el cantón Biblián, provincia del Cañar, Ecuador,

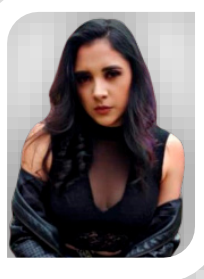
el 15 de abril del año 1993. La instrucción primaria y secundaria los realicé en la Unidad Educativa "La Providencia", Azogues; los estudios universitarios en la Universidad Católica de Cuenca (UCACUE), extensión Azogues, obteniendo el título de Licenciada en Educación, mención Educación Inicial y Parvularia; actualmente laboro como docente en la escuela de educación general básica "Héroes de Verdeloma" perteneciente al distrito 03D01, siempre buscando la superación en el campo educativo. 
Ensayo Original / Original Essay

\section{Paola Cecilia Pinos Coronel \\ e-mail: paola.pinos@psg.ucacue.edu.ec}

Nacida en la ciudad de Azogues, provincia de Cañar,

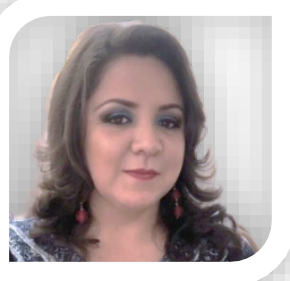

Ecuador, el 19 de noviembre del año 1988. Los estudios primarios y secundarios los realicé en la Unidad Educativa Particular "La Providencia", Azogues; los estudios universitarios en la Universidad Católica de Cuenca (UCACUE), sede Azogues, obteniendo el título de Ingeniero de Sistemas; he ejercido la labor docente durante 13 años, en la Unidad Educativa Particular "La Providencia", en las asignaturas de computación, matemáticas y emprendimiento y gestión.

El contenido de este manuscrito se difunde bajo una Licencia de Creative Commons ReconocimientoNoComercial-Compartirlgual 4.0 Internacional 